\title{
Влияние формы рассеивающих частиц на силикатную особенность $\mathrm{SiO}_{2}$
}

\author{
Д.В. Петров, Е.А. Жуюулина, А.А. Савушкин \\ ФГБУН "Крымская астрофизическая обсерватория РАН”, Научный, Крым, 298409 \\ dvp@craocrimea.ru
}

Поступила в редакцию 7 ноября 2020 г.

\begin{abstract}
Аннотация. Частицы силикатной пыли являются частью многих астрономических объектов, таких как кометы и околозвездные диски. В спектре силикаты проявляют ряд характерных эмиссионных особенностей. Обычно для изучения силикатных особенностей используется теория Ми, предполагающая, что рассеивающий объект является идеальной сферой. В данной работе мы исследовали вклад несферических частиц кварца $\left(\mathrm{SiO}_{2}\right)$ в эмиссию силикатов. Исследовано влияние отклонения от сферичности на 10-микронную силикатную особенность кварца. Показано, что отклонение от сферичности существенно изменяет как интенсивность рассеянного света, так и фактор рассеяния $Q_{s c a}$, причем с увеличением размера рассеивающей частицы это влияние возрастает. Изучены основные особенности воздействия несферичности на характеристики рассеяния как в случае вытянутых, так и сплюснутых сфероидов.
\end{abstract}

Ключевые слова: 10-микронная силикатная особенность, околозвездные диски, кометы, кварц, рассеяние света

\section{1 Введение}

Частицы силикатной пыли обнаружены в околозвездных дисках, которые окружают молодые звезды (Waelkens et al., 1997; Malfait, 1998). Наиболее распространенными видами космической пыли являются соединения кремния, железа, магния, кислорода и углерода. Физические и химические параметры силикатных пылинок, такие как размер, форма, показатель преломления и размерный параметр, являются результатом множества различных химических и физических процессов эволюции. Силикатные частицы пыли играют важную роль в понимании эволюции околозвездных дисков и процессов формирования планетных систем. Вот почему существует множество наблюдений, направленных на изучение характеристик силикатной пыли (например, Weintraub, 1989; Strom et al., 1989; Beckwith et al., 1990; Skrutskie et al., 1990).

Материалы околозвездных дисков возникают из межзвездной среды, а точнее из ядра родительского молекулярного облака. Принято считать, что пылевой состав молекулярного облака примерно совпадает с исходным пылевым составом протопланетного аккреционного диска. Небольшую разницу в составе можно объяснить испарением летучих молекулярных льдов при прохождении фронта аккреционной ударной волны. Таким образом, кислород мог взаимодействовать с атомами кремния, образуя $\mathrm{SiO}_{2}$ (кварц). Более подробное обсуждение возникновения частиц кварца в молекулярных облаках описано в обзоре Dorschner and Henning (1995).

\section{10-микронная силикатная особенность}

Наличие силикатов в околозвездных дисках проявляется в виде силикатной спектральной особенности. Наиболее заметная спектральная особенность наблюдается в окрестности длины волны 10 мкм. Именно поэтому ее часто называют 10-микронной силикатной особенностью (10- $\mu \mathrm{m}$ silicate feature) 
(Hanner et al., 1993). Эта особенность проявляется в увеличении интенсивности излучения в спектральном диапазоне от 8 до 12 мкм. Частицы силиката могут проявлять эмиссионную особенность на данных длинах волн благодаря наличию колебательных энергетических уровней связей $\mathrm{Si}-\mathrm{O}$ (Potter Jr. and Morgan, 1982; Hanner et al., 1997). Следует отметить, что данная силикатная особенность проявляется только теми частицами, температура которых более $100 \mathrm{~K}$ (Lee et al., 2013). Далее, говоря о силикатной особенности, мы подразумеваем, что температура рассеивающего объекта более $100 \mathrm{~K}$.

Эта эмиссионная особенность проявляется многими астрономическими объектами. Например, она обнаружена в излучении квазаров (Нао et al., 2005; Siebenmorgen et al., 2005). Многие кометы обладают 10-микронной силикатной особенностью. Например, кометы Mueller (1994 I = C/1993 A1) (Hanner et al., 1994), Bradfield (1987 XXIX = C/1987 P1) (Hanner et al., 1990), Hyakutake (C/1996 B2) и Hale-Bopp (C/1995 O1) (Hayward et al., 2000; Wooden et al., 1999), 1P/Halley (Bregman et al., 1987; Campins and Ryan, 1989) и Levy (1990 XX = C/1990 K1) (Lynch, 1992). Однако следует уточнить, что в формирование 10-микронной силикатной особенности комет вносят вклад лишь маленькие частицы кометной пыли, размером до 1 мкм. Это связано не с оптическими, а с термодинамическими свойствами кометной пыли - частицы большего размера просто не успевают достаточно разогреться, чтобы внести заметный вклад в данную особенность (Hanner and Bradley, 2004).

Помимо этого, два объекта Herbig $\mathrm{Ae} / \mathrm{Be}, \mathrm{LkH} \alpha 208$ и $\mathrm{LkH} \alpha$ 198, имеют характеристики излучения с максимумом около 9 мкм (Hanner and Brooke, 1998). В инфракрасном спектре некоторых звезд типа Т Тельца, полученном с помощью инфракрасного спектрографа Spitzer Space Telescope, Sargent et al. (2009) обнаружили заметные узкие эмиссионные особенности на некоторых длинах волн в спектральном диапазоне от 9 до 20 мкм, интерпретируя их как признак наличия $\mathrm{SiO}_{2}$.

Давно установлено, что основной вклад в формирование этой особенности вносит оливин, который достаточно тщательно исследован (Hanner and Bradley, 2004). В частности, в ходе лабораторных экспериментов с использованием спектрометра среднего ИК-диапазона были исследованы спектры больших (размером до 0.5 мм) частиц оливина неправильной формы в поисках 10-микронной силикатной особенности. Эта особенность была экспериментально обнаружена (Chornaya, 2020), хотя многочисленные попытки компьютерного моделирования, основанные на предположении о сферической форме рассеивателя, показывали, что при столь больших размерах частиц силикатной особенности существовать не должно (например, Hanner et al., 1987; Hage and Greenberg, 1990; Hanner et al., 1992). Единственное возможное объяснение - влияние несферичности формы рассеивателя.

Помимо оливина, в составе небесных тел есть и другие силикаты, которые могут вносить заметный вклад в эту особенность. Например, исследование кометы Wild 2 с помощью кометного пылеуловителя Stardust показывает, что кометная пыль содержит заметное количество частиц $\mathrm{SiO}_{2}$ в различных образцах оксидов (Kearsley et al., 2008). Поэтому рассмотрение вопроса о вкладе частиц кварца в 10-микронную силикатную особенность представляется достаточно важным.

Существует сравнительно мало работ, посвященных 10-микронной силикатной особенности именно кварца. В работе Henning and Meeus (2009) рассматривались частицы кварца, чей размер составлял всего лишь 0.1 мкм, т. е. был намного меньше, чем длина волны. Даже мелкие кометные частицы, вносящие заметный вклад в эмиссионную особенность, могут быть на порядок большего размера. Более того, силикаты, входящие в состав околозвездных дисков, также могут обладать намного большими размерами.

B pаботе Petrov et al. (2020) были изучены основные свойства 10-микронной силикатной особенности кварца для сферических частиц, сравнимых и превышающих длину волны. Для вычислений использовалась теория Ми (Mie, 1908), программная реализация которой является наиболее быстрой среди всех аналогичных методик расчета характеристик рассеянного света. Однако в природе сферические частицы встречаются достаточно редко. Интересно исследовать влияние отклонений от сферичности на 10-микронную силикатную особенность кварца. Изучению данного вопроса и посвящена эта работа.

\section{3 Методика расчета рассеяния несферическими частицами}

Для определения рассеивающих свойств несферических частиц потребовалось достаточно большое количество компьютерных вычислений. Потому была использована наиболее быстрая про- 
грамма для вычисления рассеивающих свойств сфероидов, разработанная Михаилом Мищенко (Mishchenko, 1991, 1993; Mishchenko, Travis, 1994). Вычислительный метод основан на методе Тматриц (Mishchenko et al., 1996) и оптимизирован для частиц, обладающих осью вращения, что максимально упрощает и ускоряет вычисления (Wielaard et al., 1997). Более того, метод Т-матриц позволяет осуществлять аналитическое усреднение характеристик рассеянного света по ориентациям рассеивающей частицы (Petrov et al., 2006).

В качестве объекта изучения использовались сфероиды (эллипсоиды вращения) с соотношением осей a/b. Здесь b- размер частицы вдоль оси вращения, a - размер оси, перпендикулярной оси вращения. Следовательно, при a/b < 1 получается сфероид, вытянутый вдоль оси вращения; при $\mathrm{a} / \mathrm{b}>1$ получается сфероид, сплюснутый вдоль оси вращения; при a/b = 1 получается сферическая частица. В данной работе были изучены частицы с соотношением осей $\mathrm{a} / \mathrm{b}=0.5, \mathrm{a} / \mathrm{b}=1.0$ и $\mathrm{a} / \mathrm{b}=$ 2.0. Характеристики рассеянного света усреднены по ориентациям рассеивающих частиц. Важно отметить, что положение эмиссионной спектральной особенности сильно зависит от размера частицы R. B случае сферы под размером частицы подразумевается радиус сферы, а в случае вытянутого и сплюснутого сфероидов - радиус сферы эквивалентного объема.

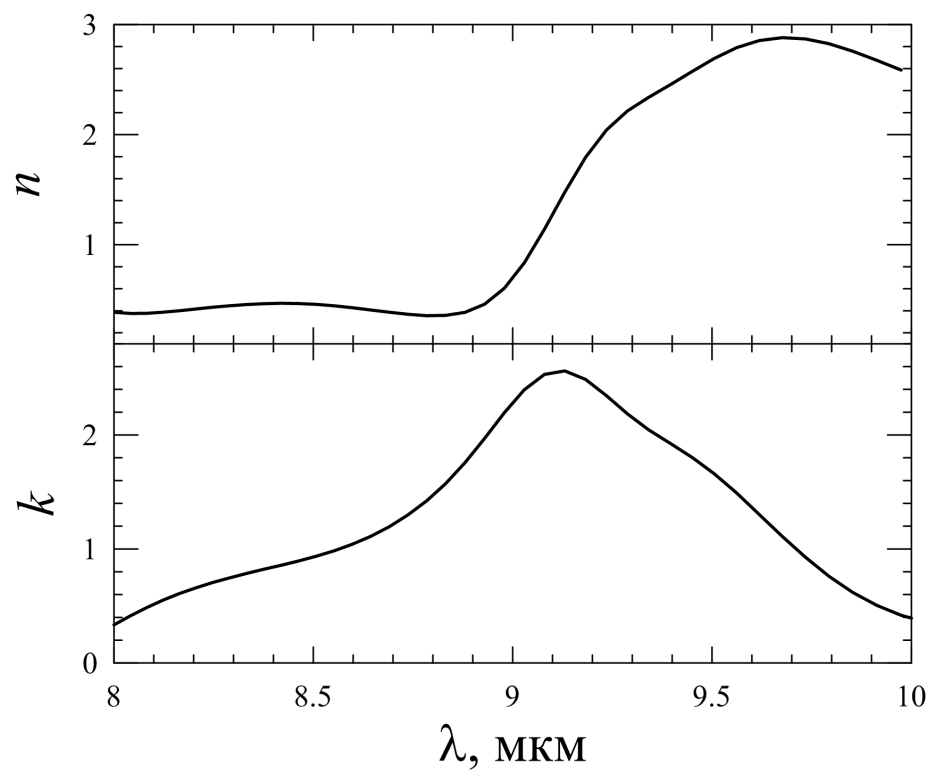

Рис. 1. Спектральная зависимость показателя преломления $\mathrm{SiO}_{2}$ (Попова и др., 1972). Верхняя панель соответствует действительной части показателя преломления, нижняя - мнимой

Основным параметром, определяющим свойства спектрально-эмиссионной особенности, является комплексный показатель преломления $m=n+i \cdot k$. Спектральная зависимость действительной и мнимой частей показателя преломления $\mathrm{SiO}_{2}$ (см. рис. 1) взята из работы (Попова и др., 1972).

\section{4 Результаты и обсуждение}

Мы рассчитали интенсивность света, рассеянного на частицах кварца различной формы, для разных длин волн и размеров рассеивающей частицы. Поскольку частицы разного размера по-разному рассеивают свет, соответствующая часть спектра была нормирована для каждого размера рассеивающей частицы - максимальное значение интенсивности света принималось равным единице. Отметим, что расчеты проводились при угле рассеяния 0 градусов (рассеяние вперед).

Рисунок 2 демонстрирует зависимость длины волны $\lambda_{\max }$, соответствующей максимуму 10-микронной силикатной особенности, от размера рассеивающей частицы. Черная линия соответствует сферической частице $(\mathrm{a} / \mathrm{b}=1)$, синяя линия - сплюснутому сфероиду $(\mathrm{a} / \mathrm{b}=2)$ и красная линия вытянутому сфероиду $(\mathrm{a} / \mathrm{b}=0.5)$. Линии обрываются на том размере рассеивающей частицы, выше которого программа Мищенко неспособна вычислять рассеивающие свойства при данном соотношении осей и показателей преломления. 
Однако даже имеющихся данных достаточно для определенных выводов. Во-первых, в случае частиц размером менее 1 мкм, вносящих максимальный вклад в 10-микронные силикатные особенности комет, положение максимума спектральной особенности смещено в область больших длин волн. Помимо этого, 10-микронная силикатная особенность вытянутого сфероида сильнее отличается от силикатной особенности сферической частицы, чем в случае сплюснутого сфероида. При этом на больших размерах любая несферичность приводит к смещению положения максимума силикатной особенности в сторону меньших длин волн.

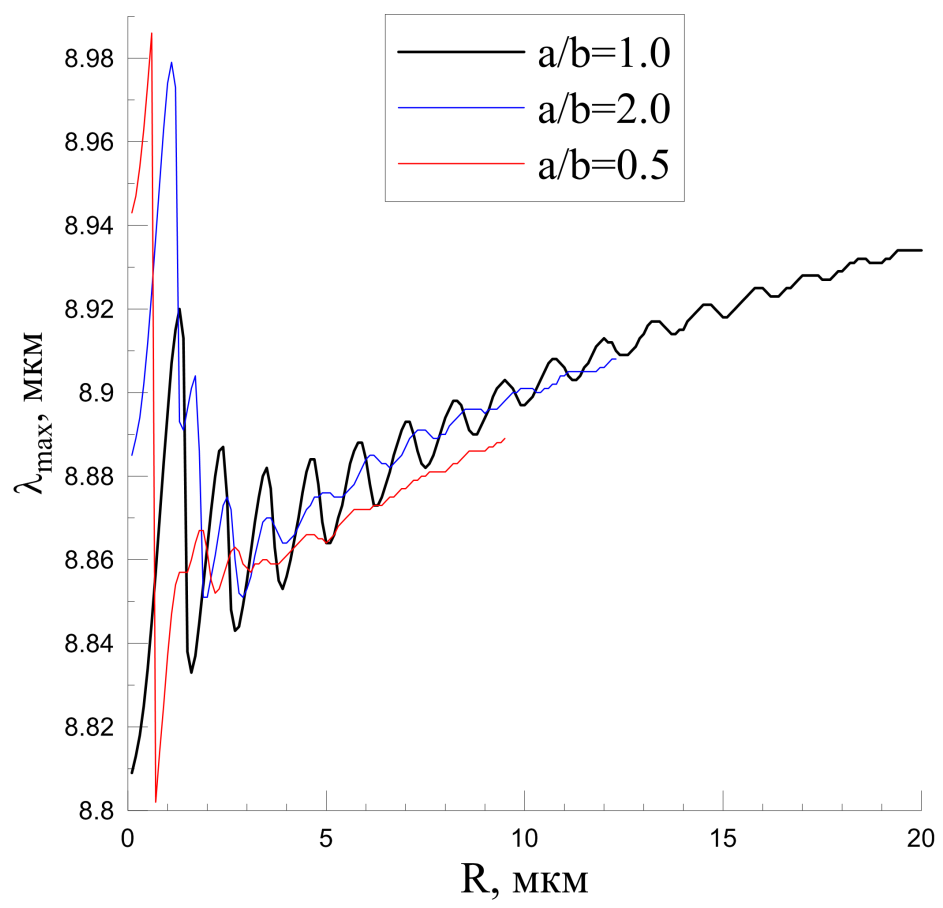

Рис. 2. Спектральное положение максимума 10-микронной силикатной особенности кварца для сферических частиц (черная линия), сплюснутых сфероидов (синяя линия) и вытянутых сфероидов (красная линия) в зависимости от размера рассеивающей частицы

Вышеописанная программа Мищенко позволяет вычислить не только интенсивность рассеянного света, но и иные характеристики рассеяния. В частности, произведение сечения рассеяния $C_{s c a}$ на величину падающего потока энергии дает полную мощность, изымаемую объектом из падающего поля за счет перерассеяния электромагнитной энергии во всех направлениях (Mishchenko et al., 2000). Все оптические сечения являются вещественными и неотрицательными величинами и имеют размерность площади. Они зависят от направления, поляризации и длины волны падающего излучения, а также от размера, морфологии, показателя преломления и ориентации рассеивающего объекта.

Гораздо нагляднее оперировать не сечением рассеяния, а фактором рассеяния $Q_{s c a}$, определенным как отношение сечения рассеяния к площади поперечного сечения объекта (Фарафонов и др., 2019):

$$
Q_{s c a}=\frac{C_{s c a}}{\pi R^{2}}
$$

Фактор рассеяния определяет, насколько эффективно перерассеивает свет единица площади рассеивающего объекта. Спектральная зависимость фактора рассеяния $Q_{s c a}$ приведена на рисунке $3 \mathrm{a}-$ в. Как и на рисунке 2 , черная линия соответствует сферической частице $(\mathrm{a} / \mathrm{b}=1)$, синяя линия - сплюснутому сфероиду $(\mathrm{a} / \mathrm{b}=2)$ и красная линия - вытянутому сфероиду $(\mathrm{a} / \mathrm{b}=0.5)$. Рисунок 3а соответствует размеру рассеивающей частицы $\mathrm{R}=3$ мкм, рис. $3 б-\mathrm{R}=6$ мкм и рис. 3 в $\mathrm{R}=9$ мкм. 

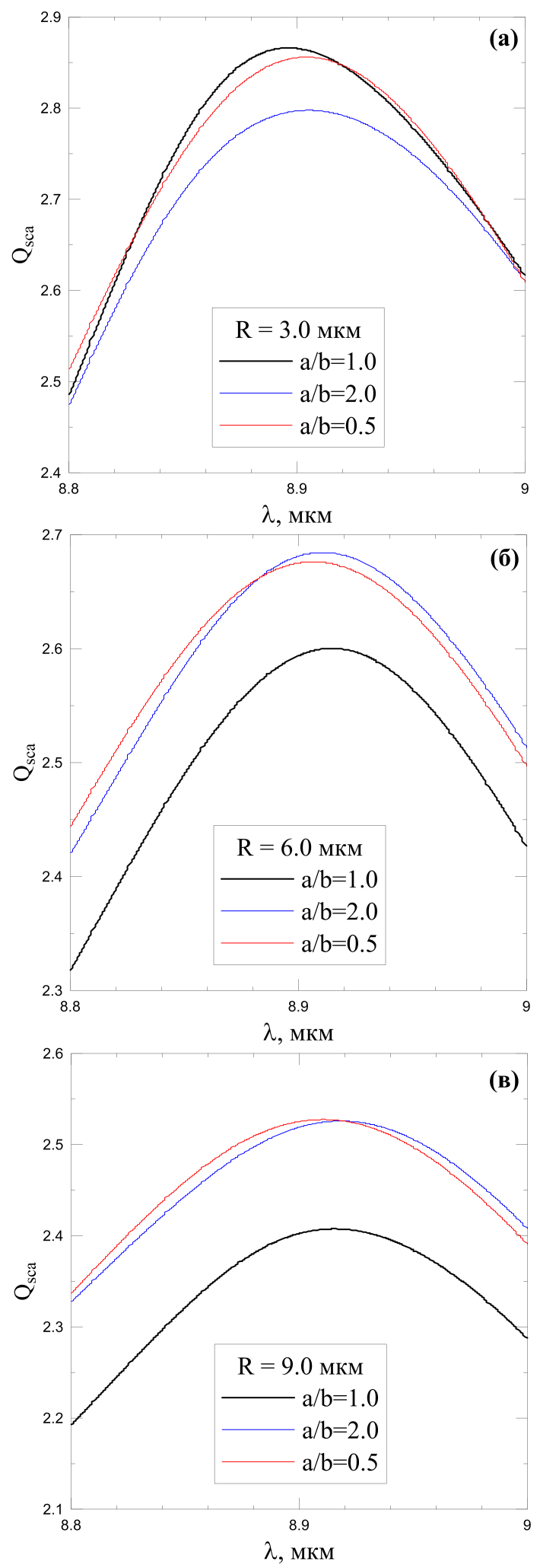

Рис. 3. Спектральная зависимость фактора рассеяния $Q_{\text {sca }} 10$-микронной силикатной особенности кварца для сферических частиц (черная линия), сплюснутых сфероидов (синяя линия) и вытянутых сфероидов (красная линия) при различных размерах рассеивающей частицы: а) $\mathrm{R}=3$ мкм; б) $\mathrm{R}=6$ мкм; в) $\mathrm{R}=9$ мкм 
Из рисунка можно сделать выводы, что эффективность рассеяния единицей площади рассеивающей частицы кварца убывает при увеличении размера частицы. В случае достаточно больших частиц (6 микрон и более) несферические частицы кварца в области 10-микронной силикатной особенности рассеивают свет в целом более эффективно, чем сферические. При этом с увеличением размера разница между несферическими и сферическими частицами возрастает.

\section{5 Выводы}

Основной вывод данной работы: учет несферичности частиц важен для изучения спектров космической пыли. Показано, что отклонение от сферичности оказывает заметное влияние на 10-микронную силикатную особенность кварца. Для частиц размером менее 1 мкм, вносящих основной вклад в 10-микронную силикатную особенность комет, положение спектральной особенности смещено в область больших длин волн. В случае относительно больших частиц, размером сравнимых или превышающих длину волны, положение спектральной особенности вытянутых сфероидных частиц кварца заметно смещено в область меньших длин волн; в случае же сплюснутых сфероидных частиц это смещение практически отсутствует. Установлено, что отклонение от сферичности оказывает существенное влияние на фактор рассеяния $Q_{s c a}$, причем с увеличением размера рассеивающей частицы это влияние возрастает.

\section{Литература}

Попова С., Толстых Т., Воробьев В., 1972. Оптика и спектроскопия. Т. 33. С. 444-445. [Popova S., Tolstykh T., Vorobev V., 1972. Optics and spectroskopy, vol. 33, pp. 444-445. (In Russ.)]

Фарафонов В.Г., Ильин В.Б., Прокопьева М.С., Тулегенов А.Р., Устимов В.И., 2019. Оптика и спектроскопия. T. 126. № 4. C. 443-449. [Farafonov V.G., Il’in V.B., Prokop'eva M.S., Tulegenov A.R., Ustimov V.I., 2019. Optics and spectroskopy, vol. 126, no. 4, pp. 443-449. (In Russ.)]

Beckwith S.V.W., Sargent A.I., Chini R.S., et al., 1990. Astron. J., vol. 99, p. 924.

Bregman J.D., Witteborn F.C., Allamandola L.J., et al., 1987. Astron. Astrophys., vol. 187, no. 1-2, pp. $616-620$.

Campins H. and Ryan E., 1989. Astrophys. J., vol. 341, pp. 1059-1066.

Chornaya E., Zakharenko A.M., Zubko E.S., et al., 2020. Icarus, vol. 350, article id. 113907.

Dorschner J. and Henning T., 1995. Astron. Astrophys. Rev., vol. 6, no. 4, pp. 271-333.

Hage J.I. and Greenberg J.M., 1990. Astrophys. J., vol. 361, pp. 251-259.

Hanner M.S., Tokunaga A.T., Golisch W.F., et al., 1987. Astron. Astrophys., vol. 187, pp. 653-660.

Hanner M.S., Newburn R.L., Gehrz R.D., et al., 1990. Astrophys. J., vol. 348, pp. 312-321.

Hanner M.S., Veeder G.J., Tokunaga A.T., 1992. Astron. J., vol. 104, pp. 386-393.

Hanner M.S., Lynch D.K., Russell R.W., 1993. Asteroids, Comets, Meteors 1993, Abstracts for the IAU Symposium 160. LPI Contribution, Houston: Lunar and Planetary Institute, vol. 810, p. 129.

Hanner M.S., Hackwell J.A., Russell R.W., et al., 1994. Icarus, vol. 112, pp. 490-495.

Hanner M.S., Gehrz R.D., Harker David E., et al., 1997. Earth Moon and Planets, vol. 79, no. 1, pp. 247264.

Hanner M.S. and Brooke T.Y., 1998. Astron. J., vol. 502, pp. 871-882.

Hanner M.S. and Bradley J.P., 2004. In Festou M.C. et al. (Eds), Comets II. Tucson: University of Arizona Press, p. 555.

Hao L., Spoon H.W.W., Sloan G.C., et al., 2005. Astrophys. J., vol. 625, no. 2, pp. L75-L78.

Hayward T.L., Hanner M.S., Sekanina Z., 2000. Astrophys. J., vol. 538, pp. 428-455.

Henning T. and Meeus G., 2009. In Garcia P.J.V. (Ed.), Physical Processes in Circumstellar Disks around Young Stars. Chicago: Univ. Chicago Press, pp. 114-148.

Kearsley A.T., Borg J., Graham G.A., et al., 2008. Meteoritics Plan. Sci., vol. 43, no. 1, pp. 41-73.

Lee J.C., Kriss G.A., Chakravorty S., et al., 2013. Mon. Not. Roy. Astron. Soc., vol. 430, no. 4, pp. 26502679.

Lynch D.K., Russell R.W., Hackwell J.A., et al., 1992. Icarus, vol. 100, pp. 197-202.

Malfait K., Waelkens C., Waters L.B.F.M., et al., 1998. Astron. Astrophys., vol. 332, pp. L25-L28.

Mie G., 1908. Annalen der Physik, vol. 330, no. 3, pp. 377-445. 
Mishchenko M.I., 1991. J. Opt. Soc. Am. A., vol. 8, pp. 871-882.

Mishchenko M.I., 1993. Appl. Opt., vol. 32, pp. 4652-4666.

Mishchenko M.I., Travis L.D., 1994. Opt. Commun., vol. 109, pp. 16-21.

Mishchenko M.I., Travis L.D., Mackowski D.W., 1996. J. Quant. Spectrosc. Radiat. Transfer, vol. 55, pp. 535-575.

Mischenko M.I., Hovenier J.W., Travis L.D., 2000. Light Scattering by Nonspherical Particles: Theory, Measurements, and Applications. Academic Press.

Petrov D., Synelnyk E., Shkuratov Y., et al., 2006. J. Quant. Spectrosc. Radiat. Transf., vol. 102, no. 1, pp. 85-110.

Petrov D., Savushkin A., Zhuzhulina E., 2020. Research Notes of the AAS, vol. 4, no. 9, p. 161.

Potter A.E. Jr. and Morgan T.H., 1982. Lunar and Planetary Science Conference Proceedings, vol. 12, pp. 703-713.

Sargent B.A., Forrest W.J., Tayrien C., et al., 2009. Astrophys. J., vol. 690, pp. 1193-1207.

Siebenmorgen R., Haas M., Krugel E., et al., 2005. Astrophys. J., vol. 436, p. L5. doi:10.1051/0004-6361:200500109

Skrutskie M.F., Dutkevitch D., Strom S.E., et al., 1990. Astron. J., vol. 99, pp. 1187-1195. doi:10.1086/115407

Strom K.M., Strom S.E., Edwards S., et al., 1989. Astron. J., vol. 97, p. 1451.

Waelkens C., Malfait K., Waters L.B.F.M., 1997. Astrophys. Space Sci., vol. 255, no. 1/2, pp. 25-33.

Weintraub D.A., Sandell G., Duncan W.D., 1989. Astrophys. J. Lett., vol. 340, p. L69.

Wielaard D.J., Mishchenko M.I., Macke A., Carlson B.E., 1997. Appl. Opt., vol. 36, pp. 4305-4313.

Wooden D.H., Harker D.E., Woodward C.E., et al., 1999. Astrophys. J., vol. 517, pp. 1034-1058. 


\title{
The shape of scattering particles influence on the $\mathrm{SiO}_{2}$ silicate feature
}

\author{
D. Petrov, E. Zhuzhulina, A. Savushkin
}

Crimean Astrophysical Observatory, Nauchny, 298409

dvp@craocrimea.ru

\begin{abstract}
Silicate dust particles are part of many astronomical objects such as comets and circumstellar discs. In the spectrum, silicates exhibit a number of characteristic silicate emission features. To study these features, Mie's theory is usually used. This theory assumes that the scattering object is an ideal sphere. In this work, we investigated the contribution of non-spherical quartz particles $\left(\mathrm{SiO}_{2}\right)$ to these features. We studied the influence of the deviation from sphericity on the 10-micron silicate feature of quartz. It is shown that the deviation from sphericity has a significant effect on both the scattered light intensity and the scattering factor $Q_{s c a}$, and this effect increases at increasing scattering particle size. The main peculiarities of the 10-micron silicate feature have been studied for both prolate and oblate spheroids.
\end{abstract}

Key words: $10-\mu \mathrm{m}$ silicate feature, circumstellar disks, comets, quartz, light scattering 